\title{
Associations between Drinking Water Source and Gut Microbiota Composition in the American Gut Project Database
}

\author{
Tiphaine Vanhaecke \\ Water Science Team, Danone Research, Palaiseau, France
}

\section{Keywords \\ Drinking water - Gut microbiota diversity - 16s rRNA gene}

\begin{abstract}
Introduction: The gut microbiome exerts a fundamental role in host physiology. Extrinsic factors such as lifestyle and diet are widely recognized as the main drivers of gut microbiota composition [1, 2]. While drinking water is among the food items consumed in the largest amount, little is known about its potential impact on gut microbiota structures [3-5]. $\mathbf{O b}$ jective: We explored the associations between plain drinking water source and gut microbiota compositions in a large microbiota-based cohort. Methods: Participants in the American Gut Project database provided fecal samples and completed health, lifestyle, and food records which included plain drinking water source (bottled, tap, filtered, or well water). Associations between drinking water source and gut microbiota were evaluated using models adjusted for anthropometric, diet, and lifestyle factors in 3,413 individuals [6]. Index of intra-individual fecal microbial diversity, inter-individual differences in composition, and taxa abundance were
\end{abstract}

estimated by $16 \mathrm{~S}$ rRNA sequencing. Results: The type of drinking water was associated with fecal microbiota composition. Drinking water source ranked among the key contributing factor explaining the gut microbiota variation both in alpha and beta diversity analyses, with effect sizes comparable to that of alcohol or diet type [6] (Fig. 1). Subjects drinking different sources of water had differences in gut microbiota signatures, as revealed by beta diversity analyses $(p<$ 0.05; Bray-Curtis dissimilarity, Weighted UniFrac distance) [6]. Subjects drinking mostly well water also had higher fecal alpha diversity than the other groups ( $p<0.05$; Faith's PD, Observed OTUs) [6]. Taxonomic differences were found in well water drinkers, with clinically important taxa, such as Bacteroides, Odoribacter, and Streptococcus being depleted and Dorea being increased as compared to the other groups [6]. Conclusions: Our results reveal that drinking water may be an important factor in shaping the gut microbiome. Future research investigating the gut microbiota in relation to environmental factors may benefit from integrating drinking water source as a covariate in the analyses.

(C) 2022 The Author(s)

Published by S. Karger AG, Basel karger@karger.com www.karger.com/anm

Karger $\stackrel{\text { ! }}{=}$

BOPEN ACCESS
(C) 2022 The Author(s)

Published by S. Karger AG, Basel

This article is licensed under the Creative Commons Attribution 4.0 International License (CC BY) (http://www.karger.com/Services/ OpenAccessLicense). Usage, derivative works and distribution are permitted provided that proper credit is given to the author and the original publisher.
Correspondence to:

Tiphaine Vanhaecke, tiphaine.vanhaecke@ danone.com 


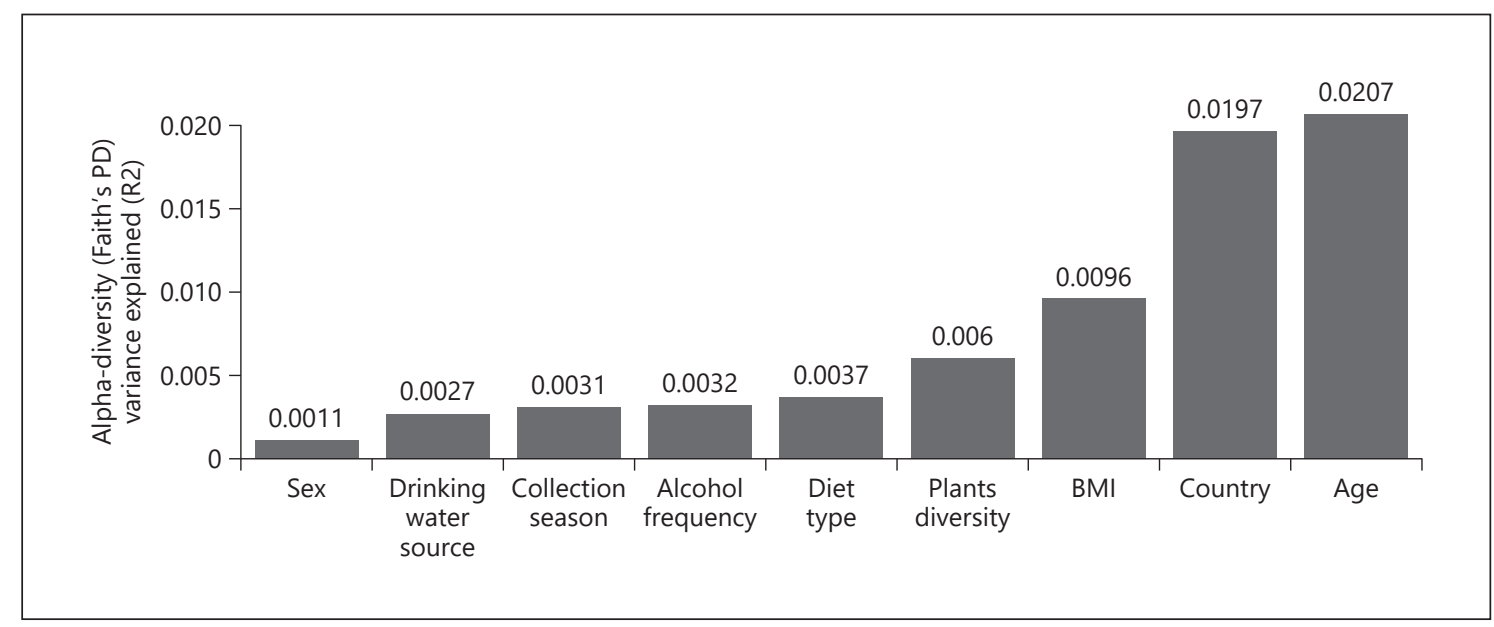

Fig. 1. Effect sizes of $\alpha$ Diversity as measured by Faith's PD. Proportions of variance captured by significant variables in model adjusted models for age, sex, BMI, infant feeding, level of education, country, collection season, exercise frequency, diet type, plant diversity (number of types of plants consumed per week), alcohol, and sugarsweetened beverage consumption. Adapted from Vanhaecke et al. [6].

\section{Statement of Ethics}

Ethical Committee approval for the collection of AGP data was obtained either from the University of Colorado Boulder Review Board (protocol No. 12-0582; December 2012-March 2015) or from the University of California Review Board, San Diego (protocol No. 141853; February 2015-present), in accordance with the Declaration of Helsinki, and all participants provided written informed consent. Return of results to participants, public deposition of de-identified data, and subsequent analyses are allowed by the IRB-approved protocol [7].

\section{Conflict of Interest Statement}

T.V. is a full-time employee of Danone Research.

\section{Funding Sources}

The author reported no funding received for this study.

\section{Data Availability Statement}

Data for this research originate from the AGP database, a selfselected citizen-scientist cohort initiated by the University of California, San Diego [7]. It contains analyses of fecal, oral, and skin samples sent by $>24,000$ volunteers worldwide. After contributing USD 99, participants receive a kit to collect their biological samples and mail them for 16S rRNA gene sequencing to establish their microbial composition. Self-reported metadata are collected through a web portal (https://microsetta.ucsd.edu/). Surveys, methodology, sampling, and laboratory test procedures have been described elsewhere [7].

\section{References}

1 Wu GD, Chen J, Hoffmann C, Bittinger K, Chen YY, Keilbaugh SA, et al. Linking longterm dietary patterns with gut microbial enterotypes. Science. 2011;334(6052):105-8.

2 Ding T, Schloss PD. Dynamics and associations of microbial community types across the human body. Nature. 2014;509(7500): 357-60.

3 Dias MF, Reis MP, Acurcio LB, Carmo AO, Diamantino CF, Motta AM, et al. Changes in mouse gut bacterial community in response to different types of drinking water. Water Res. 2018;132:79-89.
4 Jha AR, Davenport ER, Gautam Y, Bhandari D, Tandukar S, Ng KM, et al. Gut microbiome transition across a lifestyle gradient in Himalaya. PLoS Biol. 2018;16(11):e2005396.

5 Bowyer RCE, Schillereff DN, Jackson MA, Le Roy C, Wells PM, Spector TD, et al. Associations between UK tap water and gut microbiota composition suggest the gut microbiome as a potential mediator of health differences linked to water quality. Sci Total Environ. 2020;739:139697.
6 Vanhaecke T, Bretin O, Poirel M, Tap J. Drinking water source and intake are associated with distinct gut microbiota signatures in US and UK populations. J Nutr. 2021 Oct 12;nxab312. Epub ahead of print.

7 McDonald D, Hyde E, Debelius JW, Morton JT, Gonzalez A, Ackermann G, et al. American gut: an open platform for citizen science microbiome research. mSystems. 2018;3(3): e00031-18. 